\title{
A Helical Magnet Design for RHIC*
}

\author{
E. Willen, R. Gupta, A. Jain, E. Kelly, G. Morgan, J. Muratore, R. Thomas \\ Brookhaven National Laboratory, Upton, NY 11973
}

\section{Abstract}

Helical dipole magnets are required in a project for the Relativistic Heavy Ion Collider (RHIC) to control and preserve the beam polarization in order to allow the collision of polarized proton beams. Specifications are for low current superconducting magnets with a $100 \mathrm{~mm}$ coil aperture and a 4 Tesla field in which the field rotates 360 degrees over a distance of 2.4 meters. A magnet meeting the requirements has been developed that uses a small diameter cable wound into helical grooves machined into a thick-walled aluminum cylinder.

\section{INTRODUCTION}

In order to control the spin of polarized protons in the Relativistic Heavy Ion Collider (RHIC), it is necessary to use a system of dipole magnets arranged to precess and rotate the particles' spin direction. Helical magnets, in which the dipole field rotates along the axis of the magnet, offer several advantages for this purpose compared to standard, rotated magnets: minimum beam orbit excursion, orbit excursion independent of separation between magnets, and good control of spin rotation and the spin precession axis orientation. A system using only helical magnets was first proposed by Shatunov and Ptitsin [1], although others had earlier proposed systems using a combination of helical and standard dipole magnets. Studies at Brookhaven confirm the advantages of a pure helical system and that is the system now planned for RHIC [2].

In the system chosen for RHIC, two Snakes and four Rotators are required in each ring. Snakes keep both intrinsic and imperfection resonances from depolarizing the beam by flipping the spin from up/down to down/up twice on each orbit around the machine. Rotators rotate the spin to beam-parallel or beam-antiparallel for experiments. Each Snake and Rotator is made up of four, $2.4 \mathrm{~m}$ long, $100 \mathrm{~mm}$ coil aperture magnets in which the field rotates through $360^{\circ}$ over the length of the magnet. These magnets are all able to operate up to $4 \mathrm{~T}$ but depending on location, may operate at lower fields. At the entrance to the magnet the field may be either vertical or horizontal, and the sense of the helix may be either right-handed or left-handed. Four of these magnets are encased in a stainless steel tube to provide structural support and helium containment. A beam position monitor is installed at the center of each assembly. The resulting integrated cold mass is mounted into a cryostat and the assembly replaces dummy sections in the RHIC lattice. Because the field in each magnet must be individually controlled in order to achieve maximum operational flexibility, the current leads could become a significant heat load on the system; the operating current of the magnets is therefore specified at $\leq 500 \mathrm{~A}$. The design of the system of magnets is intended to minimize the variety and therefore the cost of the magnetic elements that are needed. A companion paper [3] summarizes the magnet parameters for the various Snakes and Rotators in RHIC.

\section{DESIGN CONCEPT}

The basic structure of the helical coil, shown in Fig. 1, uses a new concept: conductor in helical slots milled into thick-walled aluminum cylinders to give a $\cos \theta$ current distribution in a two-dimensional section. Two of these

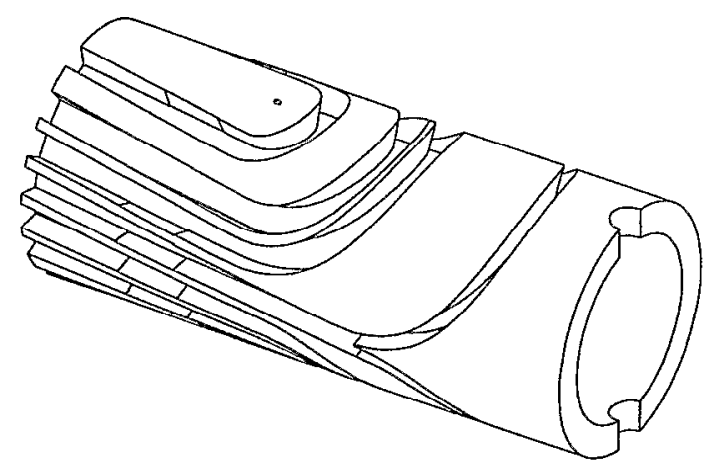

Figure 1 An isometric view of the end of the inner coil cylinder showing slots ready to accept conductor.

cylinders, concentric with one another, are assembled to give the required field of $4 \mathrm{~T}$. A cross section of the design is shown in Fig. 2.

The Lorentz forces are contained initially by the strength of the aluminum cylinders and the compressive force exerted by an overwrap of Kevlar and fiberglass/epoxy around the circumference of each coil. At high field, the cylinders distort into an elliptical shape, growing outward on the midplane. After deflecting a fraction of a millimeter, they reach the stop provided by the next tube or the iron yoke. These small elastic motions are not expected to affect the quench performance of the magnet nor to distort the field in any significant way.

\footnotetext{
- Work performed under the auspices of the US Department of Energy
} 


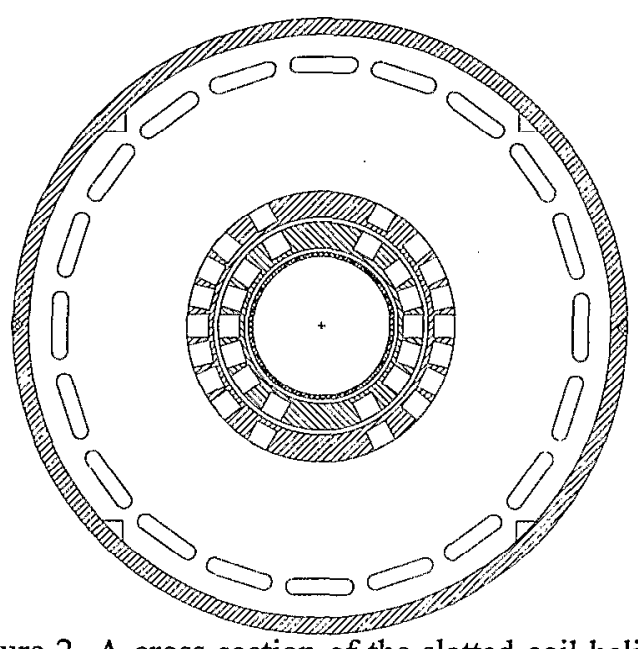

Figure 2 A cross section of the slotted coil helical magnet cold mass showing two concentric coils, the iron yoke, and the containment shell. The diameter of the iron yoke is 14 inches $(355.6 \mathrm{~mm})$.

force exerted by an overwrap of Kevlar and fiberglass/epoxy around the circumference of each coil. At high field, the cylinders distort into an elliptical shape, growing outward on the midplane. After deflecting a fraction of a millimeter, they reach the stop provided by the next tube or the iron yoke. These small elastic motions are not expected to affect the quench performance of the magnet nor to distort the field in any significant way.

\section{CONDUCTOR}

The conductor is a twisted cable made of seven strands of the $0.330 \mathrm{~mm}$ superconductor wire (Cu/non-Cu: $2.5 / 1)$ developed for the RHIC corrector program. This cable, nearly $1 \mathrm{~mm}$ in diameter, carries $314 \mathrm{~A}$ to produce a $4 \mathrm{~T}$ field in the present design. A cable is preferable to a single wire: if a break in a wire of the cable should occur, the magnet would very likely still operate satisfactorily. In addition, a cable is more flexible than an equivalent wire and therefore eases the manufacturing of the coils. The required Kapton insulation is wrapped onto the cable in the cable-manufacturing operation.

The cable is made with a 6-around- 1 geometry, which results in a cable in which the center wire is not transposed along the length of the cable. Eddy currents are generated in this wire when the magnet current is ramped. They generate heat and have a measurable effect on quench performance above a ramp rate of $2 \mathrm{~A} / \mathrm{s}$. However, since these magnets are not required to ramp with the machine, this design feature is not detrimental to the required performance.

The Kapton wrap on the cable allows space for helium inside the wrap, in direct contact with the wires. This is a desirable feature for enhancing the stability of the superconductor. It is estimated that somewhat over $10 \%$ of the volume is available for helium inside the Kapton wrap.

Fig. 3 shows the field vs. current characteristic for the magnet and the superconductor as designed.

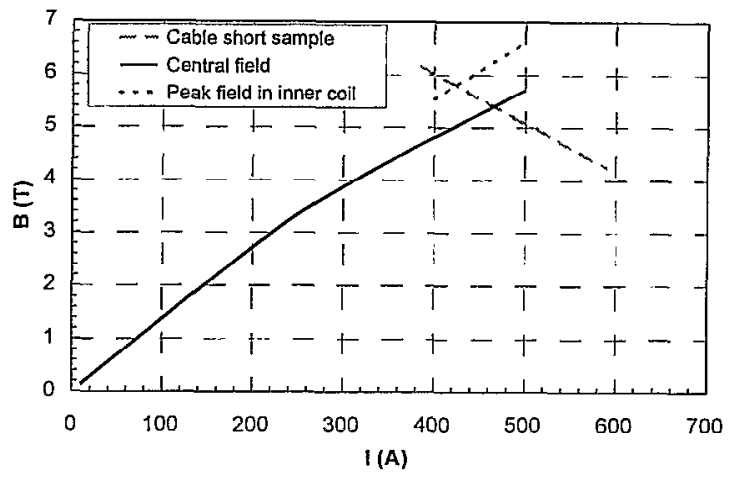

Figure 3 Field vs. current for the slotted coil helical magnet. The central field is $4 \mathrm{~T}$ at $314 \mathrm{~A}$. The peak field determines the quench current, estimated at $\sim 420 \mathrm{~A}$.

\section{MECHANICAL CONSTRUCTION}

The cable is laid in an ordered pattern into the Kapton-lined slots milled into the aluminum cylinders. A piece of fiberglass cloth impregnated with a B-stage epoxy is placed between each layer of wires in the slots. The slots continue around the ends in a layout designed to minimize undesired harmonics. When all the turns have been wound into the slots, G10 fiberglass pusher plates $3.18 \mathrm{~mm}$ thick are placed on top of the turns. Then the assembly is temporarily compressed with Kevlar that is wrapped onto the cylinder under tension. The entire assembly is next placed into an oven for curing, thereby forming a series of current blocks around the cylinder in which each wire is firmly supported in a fiberglass/epoxy matrix. After curing, any voids in the ends are filled with a mineral-loaded epoxy.

The finished diameter of a cylinder is achieved by over-wrapping each cylinder with new Kevlar strand to compress the current blocks and then with layers of fiberglass/epoxy, followed by grinding to size after curing. The two cylinders are fitted into an iron yoke supported at one end by a plate that aligns the cylinders to a fixed position. Only a slight clearance $(\sim 0.1 \mathrm{~mm})$ exists between finished cylinders. The yoke is made up of onepiece laminations having a ring of elongated holes on the outside perimeter. Tie-rods through these holes in four places provide axial restraint for the assembly (but are not required to restrain the axial Lorentz forces). Holes are also necessary for the passage of the helium coolant and the electrical buswork. The holes are designed to minimize field irregularities as the field rotates along the length of the magnet. The inner radius of the yoke is increased in the ends to reduce the peak field on the conductor. A helium containment shell is welded in place around the yoke later when the magnet is assembled into a complete helical Snake or Rotator.

A summary of mechanical parameters is given in Table 1.

\section{ELECTRICAL FEATURES}

The 16 separate windings of the two coils are connected in series at the end of the magnet. These 
Table 1 Selected mechanical parameters of the slotted coil helical magnet.

\begin{tabular}{|l|c|}
\hline \multicolumn{1}{|c|}{ Parameter } & $\begin{array}{c}\text { Value } \\
\text { Inner, Outer }\end{array}$ \\
\hline Number of cylinders & 2 \\
\hline Num of current blocks per cylinder & 7,9 \\
\hline Num of cable turns per layer & 12,12 \\
\hline Num of layers per current block & 9,9 \\
\hline Num of cable turns per block & 108,108 \\
\hline Num of cable turns per cylinder & 756,972 \\
\hline Total turns & 1728 \\
\hline Coil inner radius (mm) & $49.7,68.6$ \\
\hline Coil outer radius (mm) & $60.0,78.9$ \\
\hline Helix, magnetic length (mm) & 2400 \\
\hline Helix, rotation (deg) & 360 \\
\hline Yoke IR in straight section (mm) & 84.5 \\
\hline Yoke IR in ends (mm) & 114.4 \\
\hline Yoke outer radius (mm) & 177.8 \\
\hline
\end{tabular}

interconnections generate a small but manageable amount of heat. A low-value resistor is connected across each of the windings for protection against excessive temperature and voltage during a quench. Resistors provide a current

bypass as coil resistance builds following a quench. Without these resistors, a quench in a winding could absorb too much of the stored energy of the magnet and possibly lead to conductor damage. Since these magnets do not have a ramp rate requirement, resistors are the best choice---diodes could be used if there were a need to ramp the magnets during operation. The stored energy of the magnet at $4 \mathrm{~T}$ is $240 \mathrm{~kJ}$ ( $2.4 \mathrm{~m}$ effective length) and the inductance is $4.8 \mathrm{H}$.

\section{PROTOTYPE MAGNET \& TEST RESULTS}

A half-length prototype magnet of the slotted coil design was built and tested at Brookhaven. This magnet followed an earlier coil prototype that was built to test the concept and to evaluate the requirements of this novel coil design. The windings in the prototype magnet were placed by hand, a laborious process because of the tight dimensions of the slots; this task will be automated for production magnets. Other features of the prototype were close to those intended for production magnets.

The quench performance of the prototype magnet was excellent, as shown in Fig. 4. After two low quenches, believed to have originated in inadequately supported leads, the magnet quenched only at the level expected from prior tests on the cable. A shift in dewar temperature, and a variation in ramp rate, both moved the quench current in the expected direction---independent confirmation that the magnet was operating at the short sample limit. The current for all four quenches at the nominal test temperature $(4.35 \mathrm{~K})$ and ramp rate $(0.1 \mathrm{~A} / \mathrm{s})$ was $398 \mathrm{~A}(4.8 \mathrm{~T})$. At $4.6 \mathrm{~K}$, the magnet quenched at 378 A. Not shown are several quenches with a dual current configuration powering the coils that reached $5.0 \mathrm{~T}$, indicating that the design is not mechanically limited.
Detailed field measurements using a Hall probe system are in progress.

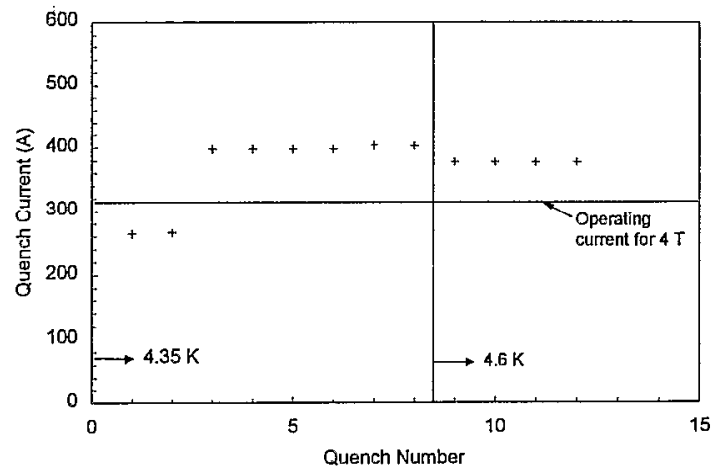

Figure 4 Quench performance of the magnet. Following two low quenches believed to have originated in the leads, the magnet quenched on a stable plateau following ramps at $0.1 \mathrm{~A} / \mathrm{s}$. Quenches 7 and 8 were slightly higher $(6 \mathrm{~A})$ because of the higher ramp rate used $(1 \mathrm{~A} / \mathrm{s})$.

Measurements of temperature rise during quench (maximum recorded $490^{\circ} \mathrm{C}$ ) on the earlier coil prototype indicated tolerable limits.

\section{Summary}

The test results confirm the adequacy of the slotted coil design. A full-length model using a slightly modified turn layout to adjust the field harmonics will be built in the coming year. The coils in this model will be fabricated with an automated wiring machine, currently under construction. Some additional tests in the next model are needed to measure the temperature rise in the final coil during quench. The design provides a field margin of $\sim 20 \%$ under operating conditions in RHIC.

\section{Acknowledgements}

The authors thank Mike Harrison for his support and encouragement of this development effort. We also thank T. Roser and M. Syphers for their continued interest and support.

\section{References}

[1] V. I. Ptitsin and Y. M. Shatunov, Helical Spin Rotators and Snakes, Proc. Third Workshop on Siberian Snakes and Rotators (A. Luccio and T. Roser, Eds.) Upton, NY, Sept. 12-13, 1994, Brookhaven National Laboratory Report BNL-52453.

[2] Preliminary Design Report, Polarized Proton Collider at RHIC, Brookhaven National Laboratory, January 28, 1997.

[3] M. Syphers et al., Helical Dipole Magnets for Polarized Protons in RHIC, Proc. PAC97, Vancouver, BC, May, 1997. 